\title{
Influence of Initial Vorticity Distribution on Axisymmetric Vortex Breakdown and Reconnection
}

\author{
Larry A. Young \\ NASA Ames Research Center, Moffett Field, CA, 94035-1000
}

\begin{abstract}
An analytical treatment has been developed to study some of the axisymmetric vortex breakdown and reconnection fluid dynamic processes underlying bodyvortex interactions that are frequently manifested in rotorcraft and propellerdriven fixed-wing aircraft wakes. In particular, the presence of negative vorticity in the inner core of a vortex filament (one example of which is examined in this paper) subsequent to "cutting" by a solid body has a profound influence on the vortex reconnection, leading to analog flow behavior similar to vortex breakdown phenomena described in the literature. Initial vorticity distributions (three specific examples which are examined) without an inner core of negative vorticity do not exhibit vortex breakdown and instead manifest diffusion-like properties while undergoing vortex reconnection. Though this work focuses on laminar vortical flow, this work is anticipated to provide valuable insight into rotary-wing aerodynamics as well as other types of vortical flow phenomena.
\end{abstract}

\section{Nomenclature}

$\mathrm{r} \quad$ Radial coordinate, origin at filament axis, $\mathrm{m}$

$r^{*} \quad$ Nondimensional radial coordinate, $\mathrm{r}^{*}=\mathrm{r} / \mathrm{s}$

$r_{o} \quad$ Spherical finite-volume source terms' "effective" initial radius, $\mathrm{m}$

$r_{c 0} \quad$ "Finite core" vortex filament core size radius (at time equal to zero), $\mathrm{m}$

$\operatorname{Re} \quad$ Vortex Reynolds number, $\operatorname{Re}=\gamma / v$

$\mathrm{s} \quad$ Axial distance of filament breakpoint (time equal zero) from origin, $\mathrm{m}$

$\mathrm{t} \quad$ Time, sec

$t^{*} \quad$ Nondimensional time parameter, $t^{*}=v t / s^{2}$

V Velocity vector, cylindrical coordinates, $\mathbf{V}=\left[\begin{array}{lll}v_{r} & v_{\theta} & v_{z}\end{array}\right], \mathrm{m} / \mathrm{sec}$

$V_{p} \quad$ Finite core vortex axial flow "wave front" propagation velocity, $\mathrm{m} / \mathrm{sec}$

$\mathrm{z} \quad$ Axial (along vortex filament axis) coordinate, origin at intersection of filament segments' plane of symmetry and filament axis, $m$

$\mathrm{z}^{*} \quad$ Nondimensional axial coordinate, $\mathrm{z}^{*}=\mathrm{z} / \mathrm{s}$

$\gamma \quad$ Vortex filament initial circulation strength, $\mathrm{m}^{2} / \mathrm{sec}$

$\Gamma \quad$ Vortex circulation, $\mathrm{m}^{2} / \mathrm{sec}$

$v \quad$ Kinematic viscosity, $\mathrm{m}^{2} / \mathrm{sec}$

$\theta \quad$ Angular coordinate, radians

$\omega \quad$ Vorticity vector, $\omega=\left[\begin{array}{lll}\omega_{r} & \omega_{\theta} & \omega_{z}\end{array}\right], 1 / \mathrm{sec}$

$\psi \quad$ Axisymmetric flow stream function

\footnotetext{
${ }^{1}$ Aerospace Engineer, Aeromechanics Branch, Flight Vehicle Research and Technology Division, Mail Stop 243-12, AIAA Associate Fellow.
} 


\section{Introduction}

$\mathrm{V}$ ORTEX filament "cutting" by solid bodies occurs in a number of real world examples for rotary-wing aircraft. These examples include blade vortex interactions where vortices shed from one rotor blade collide with another blade, trailed tip vortex interactions/collisions with a helicopter airframe/tailboom in hover and low-speed flight, or, alternatively, proprotor trailed tip vortex collisions with wing surfaces for tiltrotor aircraft in airplane-mode (Fig. 1). The time (wake age) evolution of rotor or propeller wake trailed vortex filaments can have profound implications for rotary-wing performance, acoustics, and aerial vehicle aerodynamics. With the recent renewed emphasis on rotary-wing aircraft with multiple rotors (such as high-speed coaxial aircraft and quad-tiltrotors among other vehicle configurations), the fundamental understanding of body-vortex interaction fluid dynamics is becoming increasingly important. Though tremendous progress has been made with respect to theoretical and computational fluid dynamic studies, the full implications of unsteady vortical flow phenomena are still not well understood in the rotorcraft community. Therefore, body-vortex interaction is still very much an active area for research for rotorcraft. From an experimental perspective, only a limited amount of quantitative information exists in the literature. References 1-3, for example, provide qualitative discussion regarding the vortex filament "cutting" as manifested in the form of blade vortex interactions. References 4-7 provide similar qualitative discussion of vortex and fuselage/airframe/wing interactions. Additionally, a plethora of anecdotal evidence exists in form of observations and images of rotor wakes from various aircraft where the rotor helical vortices have been documented, usually by natural condensation in the air. But these images, though oftentimes visually stunning, e.g. Ref. 8, are not particularly useful in tackling the body-vortex interaction, as well as the larger rotor/airframe interactional aerodynamics, problem.
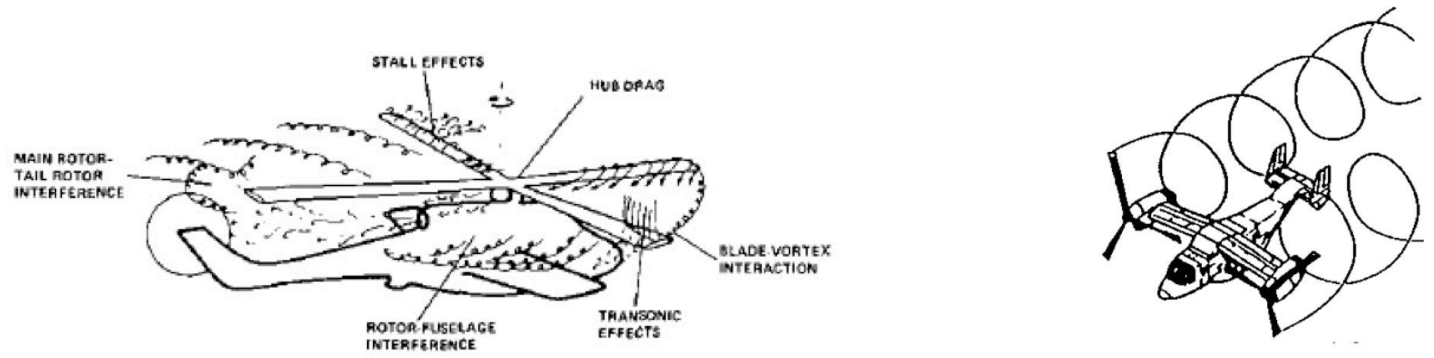

Fig. 1. Various Representative Body-Vortex Interactions; potentially including "cutting" of vortex filaments (from Ref. 9)

Very little fundamental, including theoretical, work to date has been performed examining the postcutting/collision unsteady fluid dynamics of vortex/solid body interactions. Most work has either concentrated on the vortex/body interaction process itself (see Ref. 10) or, alternatively, has looked at vortex-on-vortex interactions. A related topic is the study of ring vortices during vortex/vortex and vortex/body interactions; see Ref. 11, for example. Though flow visualization and measurement techniques are sufficiently maturing so as to examine in detail the rotary-wing/airframe vortex filament collision process - see, in particular, Refs. 4, 5, and 7 - it is clear that current rotor wake theoretical analysis is inadequate to accurately model the vortex breakdown or reconnection processes subsequent to body/vortex interaction. This work summarizes a new analysis approach to study these vortical flow processes, postfilament-cutting, during body/vortex interaction.

Analytical descriptions of columnar vortex filament reconnection and breakdown phenomena -subsequent to "cutting" or, rather, collision with a solid body -- were derived for four distinct and different initial vorticity distributions: instantaneous, uniform, parabolic, and dual-core (inner core with negative vorticity). Though all four initial vorticity distributions yielded several flow features in common with each other, there were also a number of flow phenomena that were unique to only a subset of the distributions studied. In particular, the dual-core model exhibited interesting vortical flow characteristics not only with regards to vortex filament reconnection but also manifested flow behavior analogous to that observed and 
(numerically) predicted for axisymmetric vortex breakdown. Figure 2 illustrates the three initial vorticity distributions studied.

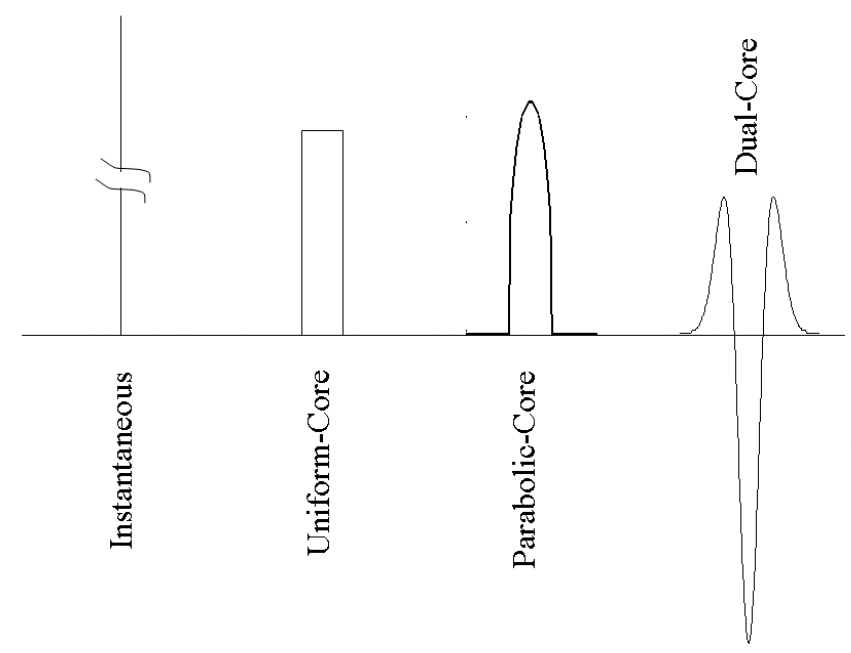

Fig. 2. Initial Vorticity Distributions Studied in Ref. 12: (a) uniform, (b) slightly parabolic, and (c) dual-core models

Figure 3 illustrates the point that there is going to be varying degrees of vortex filament "breakage" (in form of filament distortion and separation of "breakpoints") depending on the severity of the body/vortex interaction. Perpendicular collisions of vortex filaments with thin solid bodies will tend to minimize the separation distance between the filament segment breakpoints as well as minimize the filament distortion. Therefore, collision with thin solid bodies will tend to result in the "reconnection" of the vortex filaments. Conversely, interactions/collisions with bluff bodies will tend to result in large "breakpoint" separation distances and considerable filament segment distortion -- therefore, most likely resulting in vortex "breakdown."

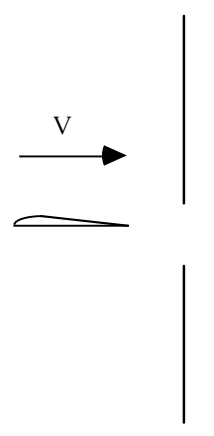

Collision with Thin Body

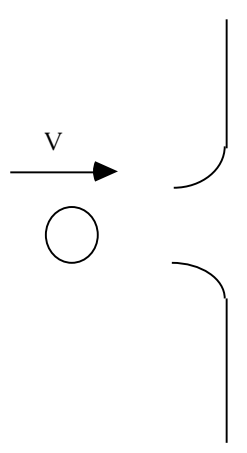

Collision with Bluff Body

Fig. 3. Hypothetical Vortex Filament Distortion/Breakage after Collision with Solid Bodies

Undoubtedly, at this point of the discussion, while discussing "cut" or "broken" vortex filament segments, concern might be raised that the proposed analysis is perhaps in violation of Kelvin's Theorem regarding the constancy of vortex circulation over (and the necessity of) closed paths. Or otherwise stated: "An important consequence of the (Kelvin) theorem is that a vortex-line cannot begin or end at any point in the interior of the fluid. Any vortex-lines which exist must either form closed curves, or else traverse the fluid, beginning and ending on its boundaries." (Ref. 13). However, as has also been pointed out by many 
others (e.g. Ref. 14), Kelvin's Theorem only holds absolutely true for inviscid flow. Further, the proposed analytical study of "cut" or "broken" vortex filament segments can be considered to fall into the same general category of gedanken ("thought") investigation as the pioneering work of Klein's Kaffeelöffel experiment (Ref. 15), wherein a plate set in motion in a fluid is "dissolved" so as to form and, subsequently, thereby leave behind a vortex sheet. This work will, of course, be shown to be consistent with all applicable governing fluid dynamic equations, as well as describing physically relevant vortical flow phenomena.

Figure 4 illustrates an idealized version of the vortex filament in the intermediate process of vortex reconnection/breakdown, post-collision. Distortion of the filament as to deflection of the filament axis laterally from its undisturbed orientation is neglected. Initial axial flow (along the filament axis) is assumed to be zero as a result of the collision process: the filament, as it wraps around the solid body, will have to adhere to the no-flow boundary constraint.

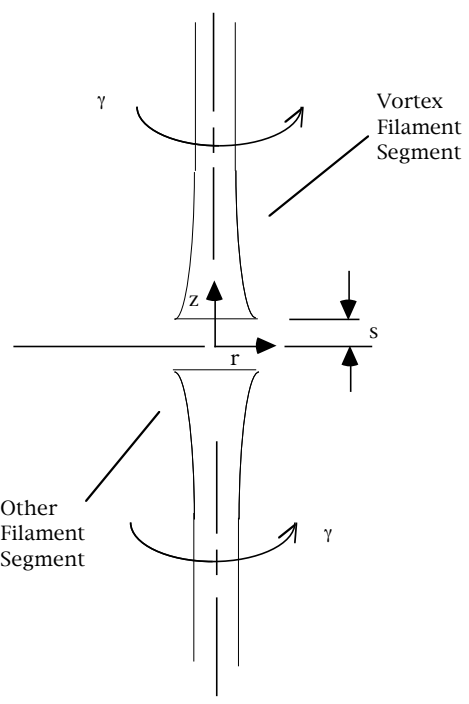

Fig. 4. Vortex Geometry Description

Figure 5 illustrates the evolution of the vortex core radius iso-surfaces with time, based on the predictions for dual-core (and parabolic) vortex filaments discussed later in the paper. The core size distribution is one means by which the vortex reconnection can be observed. The increase in tangential velocity momentum in the intermediate region between the vortex breakpoints with increasing nondimensional time can be clearly seen in Fig.5. Additionally, there is a localized increase in core size (relative to the "far-field" core size as $z^{*} \rightarrow \infty$ ) at the breakpoints. The temporal variation in core size in the immediate vicinity of the vortex breakpoints seems to grow, or fill-in, much faster for the parabolic initial vorticity distribution than for the dual-core distribution. Curiously, two of the initial vorticity distributions - the instantaneous and uniform-core distributions - do not exhibit a spatial (versus temporal) variation in vortex core size; instead in both these cases the vortex core contour is a constant diameter cylindrical surface. 

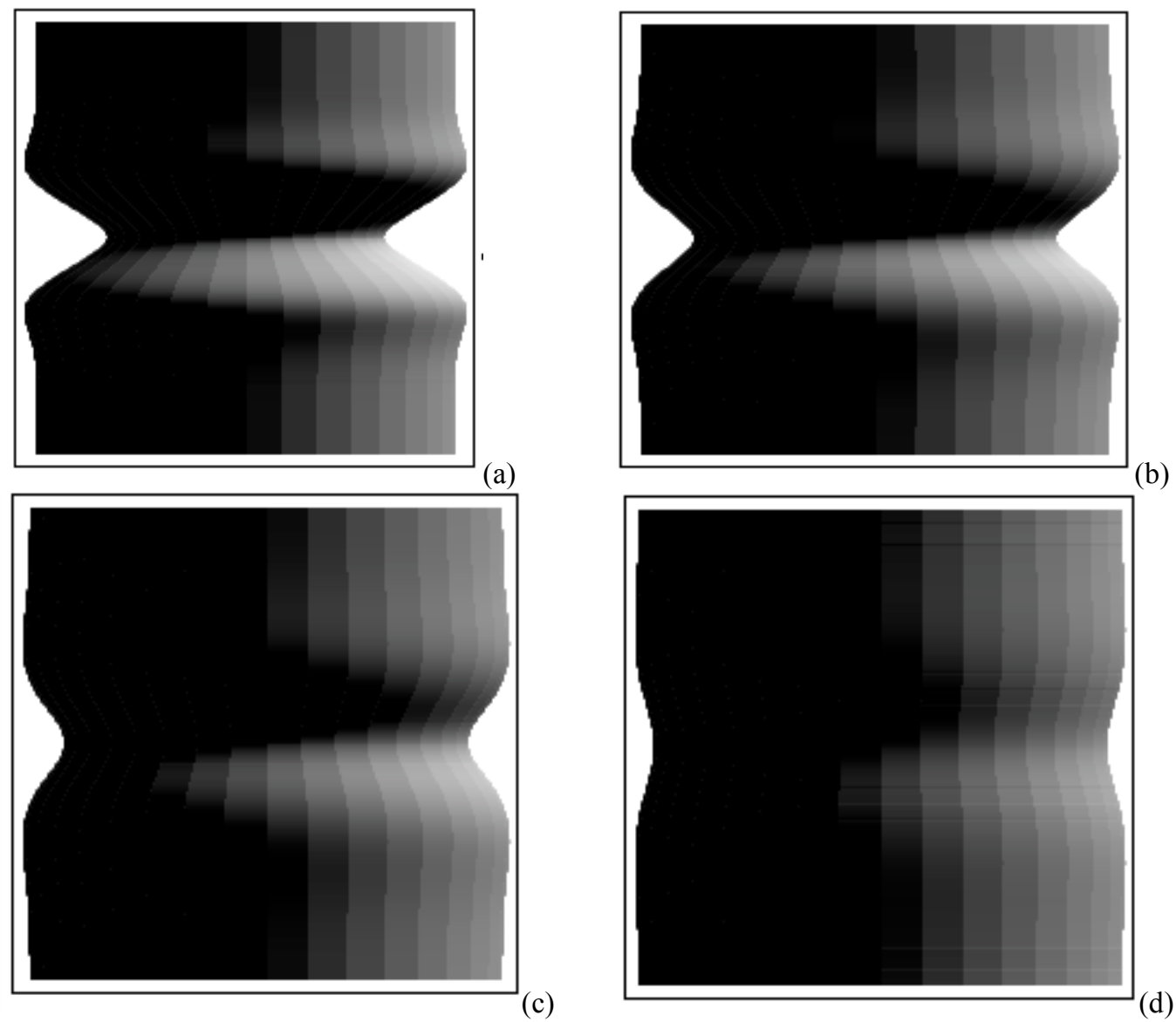

Fig. 5. Representative Trends as to "Cut" Vortex Core Evolution with Time for Dual-Core and Parabolic Distributions: (a) $t^{*}=0.12$, (b) $t^{*}=0.2$, (c) $t^{*}=0.3$, and (d) $t^{*}=0.5$

Table 1 presents a high level assessment of some of the flow phenomenology of the four different initial vorticity distributions studied. The hallmark flow features for vortex reconnection appear to be: the existence of axial flow "wave fronts" propagating away from the vortex filament "breakpoints" along the filament segment axes; "funnel-like" steam function contours in the outer flow (outside of the vortex core); and, consistent with the stream function predictions, the observation of spiral streakline, and particle trace, flow patterns also in the outer flow of the vortex filaments. These flow features are exhibited in the predictions made for all four initial vorticity distributions studied. Other noteworthy flow features that can be manifested as a part of the vortex reconnection process include: observation of complex and/or secondary flow structures in the vortex enstrophy contours at, or about, the vortex filament breakpoints early during the vortex reconnection process and spatial (versus temporal) variation of the vortex core radius. And, yet, these two additional flow features were not universally manifested by the initial vorticity distributions studied. Uniquely, the appearance of vortex breakdown phenomena, and secondary features in the circulation distribution in the intermediate region between vortex breakpoints, seems to only occur when there is negative vorticity in the inner core of the initial vorticity distribution (or, as yet to be established by future study, at least a region of reduced vorticity strength in the center of the vortex filament may be required). Finally, it should be noted that the analytical solutions for the finite-core vortices also provide valuable insight into unsteady columnar (i.e. $v_{\theta}$ is only a function of $r$ and $t$ ) vortex flow behavior for the special case of $s=0$; in this regards, the work summarized in this paper is a valuable addition to the modest body of theoretical investigations into unsteady viscous vortex models. 
Table 1 - Examples of Vortex Reconnection Phenomenology

\begin{tabular}{|l|c|c|c|c|}
\hline & Instantaneous & Uniform-Core & Parabolic-Core & Dual-Core \\
\hline $\begin{array}{l}\text { Axial "Wave Fronts" Propagating from Vortex } \\
\text { Breakpoints }\end{array}$ & $\mathrm{X}\left(V_{P} \rightarrow \infty\right)$ & $\mathrm{X}$ & $\mathrm{X}$ & $\mathrm{X}$ \\
\hline $\begin{array}{l}\text { "Funnel-Like" Stream Function Contours } \\
\text { (Outer Flow) }\end{array}$ & $\mathrm{X}$ & $\mathrm{X}$ & $\mathrm{X}$ & $\mathrm{X}$ \\
\hline $\begin{array}{l}\text { Spiral (Streakline and Particle-Trace) Flow } \\
\text { Patterns (Outer Flow) }\end{array}$ & $\mathrm{X}$ & $\mathrm{X}$ & $\mathrm{X}$ & $\mathrm{X}$ \\
\hline $\begin{array}{l}\text { Complex and/or Secondary Flow Structures in } \\
\text { Enstrophy Contours }\end{array}$ & & $\mathrm{X}$ & $\mathrm{X}$ & $\mathrm{X}$ \\
\hline $\begin{array}{l}\text { Spatial (versus Temporal) Variation of Vortex } \\
\text { Core Radius }\end{array}$ & & & $\mathrm{X}$ & $\mathrm{X}$ \\
\hline $\begin{array}{l}\text { Vortex Breakdown Bubbles } \\
\text { Secondary Features in Circulation "Diffusion" } \\
\text { into Intermediate Region }\end{array}$ & & & & $\mathrm{X}$ \\
\hline
\end{tabular}

The various types of flow phenomenon identified in Table 1 will be discussed in further detail later in the Results section. The paper now proceeds, though, with a discussion of the theoretical underpinning of the analysis presented - an approach by which the Helmholtz viscous vortical flow equation is reduced to the classic heat conduction equation.

\section{A New Class of Flow Problem}

A new class of flow problem has been identified that not only encompasses the "moving boundary" class of problems (including the classic Lamb-Oseen laminar two-dimensional vortex) but the vortex reconnection problem as well. Key to this new class of flow problem is the assumption that the axial and radial velocity components, $\mathrm{v}_{\mathrm{z}}$ and $\mathrm{v}_{\mathrm{r}}$, are not merely equal to zero (as in the case of the Lamb-Oseen vortex) but the radial velocity is instead proportional to the tangential velocity gradient, e.g.

$$
v_{r} \propto \frac{\partial v_{\theta}}{\partial z}
$$

Which dictates, in turn, that

$$
v_{z} \propto-\left(\frac{\partial v_{\theta}}{\partial r}+\frac{v_{\theta}}{r}\right)
$$

For such a class of flow problems (subject to Eqs. 1-2) the convective acceleration terms cancel out the vortex stretching terms and thereby achieving the objective of reducing the Helmholtz equation for laminar vortical flow (Eq. 3) to the unsteady heat conduction equation (Eq. 4).

$$
\begin{gathered}
\frac{D \omega}{D t}=(\omega \cdot \nabla) \mathbf{V}+v \nabla^{2} \omega \\
\frac{\partial \omega_{z}}{\partial t}=v\left\{\frac{\partial^{2} \omega_{z}}{\partial r^{2}}+\frac{1}{r} \frac{\partial \omega_{z}}{\partial r}+\frac{\partial^{2} \omega_{z}}{\partial z^{2}}\right\}
\end{gathered}
$$


Note that in the above the term $D \omega / D t$ is the particle derivative for the vorticity. And, further, the vorticity components can be defined by

$$
\boldsymbol{\omega}=\operatorname{cur} l \mathbf{V}=\nabla \times \mathbf{V}
$$

Subject to the following constraint

$$
\operatorname{div} \omega \equiv \nabla \cdot \omega=\mathbf{0}
$$

The result is the employment of length-scale factors to solve for the various three-dimensional components of the vorticity and velocity. These length-scale factors are dependent on whether or not the inherent functionality (in terms of source functions) is axially symmetrical or non-symmetric. Requiring that

$$
v_{r} \frac{\partial \omega_{z}}{\partial r}+v_{z} \frac{\partial \omega_{z}}{\partial z}=\omega_{r} \frac{\partial v_{z}}{\partial r}+\omega_{z} \frac{\partial v_{z}}{\partial z}
$$

The following formulation of length-scales holds. Let the axial and radial vorticity analytic functions be expressed as a summation of $\mathrm{N}$ source terms arranged in spatial distribution.

$$
\omega_{r}=\sum_{i=1}^{N} \omega_{r_{i}} \quad \text { And } \quad \omega_{z}=\sum_{i=1}^{N} \omega_{z_{i}}
$$

Next, assume that a length-scale can be applied to each individual source term such that

$$
v_{r}=-\sum_{i=1}^{N} \ell_{i} \omega_{r_{i}} \quad \text { And } \quad v_{z}=-\sum_{i=1}^{N} \ell_{i} \omega_{z_{i}}
$$

Making the above substitution, the following demonstrates that the nonlinear terms in the laminar axisymmetric version of the unsteady Helmholtz equation cancel out. First, note that

$$
\begin{gathered}
v_{r} \frac{\partial \omega_{z}}{\partial r}+v_{z} \frac{\partial \omega_{z}}{\partial z}=\omega_{r} \frac{\partial v_{z}}{\partial r}+\omega_{z} \frac{\partial v_{z}}{\partial z} \\
\downarrow \\
-\sum_{i=1}^{N} \ell_{i} \omega_{r_{i}} \cdot \frac{\partial}{\partial r}\left(\sum_{i=1}^{N} \omega_{z_{i}}\right)-\sum_{i=1}^{N} \ell_{i} \omega_{z_{i}} \cdot \frac{\partial}{\partial z}\left(\sum_{i=1}^{N} \omega_{z_{i}}\right)= \\
\sum_{i=1}^{N} \omega_{r_{i}} \cdot \frac{\partial}{\partial r}\left(-\sum_{i=1}^{N} \ell_{i} \omega_{z_{i}}\right)+\sum_{i=1}^{N} \omega_{z_{i}} \cdot \frac{\partial}{\partial z}\left(-\sum_{i=1}^{N} \ell_{i} \omega_{z_{i}}\right)
\end{gathered}
$$

Then, if one assumes that a single common length-scale (for the non-symmetric case) can be applied, where

$$
\ell_{N}=\ell_{N-1}=\ldots=\ell_{2}=\ell_{1}=\ell
$$


It can be readily seen from the above that

$$
\begin{gathered}
-\sum_{i=1}^{N} \ell_{i} \omega_{r_{i}} \cdot \frac{\partial}{\partial r}\left(\sum_{i=1}^{N} \omega_{z_{i}}\right)-\sum_{i=1}^{N} \ell_{i} \omega_{z_{i}} \cdot \frac{\partial}{\partial z}\left(\sum_{i=1}^{N} \omega_{z_{i}}\right)= \\
\sum_{i=1}^{N} \omega_{r_{i}} \cdot \frac{\partial}{\partial r}\left(-\sum_{i=1}^{N} \ell_{i} \omega_{z_{i}}\right)+\sum_{i=1}^{N} \omega_{z_{i}} \cdot \frac{\partial}{\partial z}\left(-\sum_{i=1}^{N} \ell_{i} \omega_{z_{i}}\right) \\
\downarrow \\
\omega_{r} \frac{\partial \omega_{z}}{\partial r}+\omega_{z} \frac{\partial \omega_{z}}{\partial z}=\omega_{r} \frac{\partial \omega_{z}}{\partial r}+\omega_{z} \frac{\partial \omega_{z}}{\partial z}
\end{gathered}
$$

The above (Eqs. 7-12), therefore, validates the utility of vorticity/velocity length-scale factors to reduce the Helmholtz equation to the heat conduction equation. In particular, it demonstrates that superposition of elemental functions representing the vorticity is possible as long as the length scale factor is uniformly applied to all elemental (source) terms. Therefore, this also validates the intended use of semicontinuous distributions of point and finite-core source terms to model the cut, or broken, vortex filaments in the reconnection problem.

Note, though, that when spanwise symmetry is preserved (as is the case for the vortex filament with two breakpoints in close proximity to each other), then there are very special implications as to the lengthscales employed for, and applied to the analytic functions describing, the vorticity distribution). Assume, first, that the equation describing the vorticity distribution can be decomposed into two elemental functions, such that

$$
\omega_{z}=\omega_{z A}+\omega_{z B}
$$

Where these two elemental functions can be defined (to the detail required) as follows:

For $t \rightarrow 0$
$\omega_{z A}=0$
When
$z \leq 0$
$\omega_{z B}=0$
When
$z \geq 0$

And for $t \rightarrow \infty$
$\omega_{z A} \neq 0$
When
$z \leq 0$
$\omega_{z B} \neq 0$
When
$z \geq 0$

Further the vorticity symmetry condition

$$
\left.\omega_{z}\right|_{z=a}=\left.\omega_{z}\right|_{z=-a}
$$

Implies the following elemental function symmetry requirements (given the above definitions of $\omega_{z A}$ and $\left.\omega_{z B}\right)$

$$
\left.\omega_{z A}\right|_{z=a}=\left.\omega_{z B}\right|_{z=-a}
$$

And

$$
\left.\omega_{z A}\right|_{z=-a}=\left.\omega_{z B}\right|_{z=a}
$$


Given axial flow symmetry considerations

$$
\left.v_{z}\right|_{z=a}=-\left.v_{z}\right|_{z=-a}
$$

And assuming that the two length-scales $\left(\ell_{A}\right.$ and $\left.\ell_{B}\right)$ need be applied to relate velocity to vorticity such that

$$
v_{z}=-\ell_{A} \omega_{z A}-\ell_{B} \omega_{z B}
$$

This, in turn, dictates that

$$
\begin{gathered}
\left.v_{z}\right|_{z=a}=-\left.v_{z}\right|_{z=-a} \\
\downarrow \\
\left.\left(-\ell_{A} \omega_{z A}-\ell_{B} \omega_{z B}\right)\right|_{z=a}=-\left.\left(-\ell_{A} \omega_{z A}-\ell_{B} \omega_{z B}\right)\right|_{z=-a}
\end{gathered}
$$

Substituting the above elemental function symmetry requirements $\left(\left.\omega_{z A}\right|_{z=a}=\left.\omega_{z B}\right|_{z=-a} \quad\right.$ and $\left.\omega_{z A}\right|_{z=-a}=\left.\omega_{z B}\right|_{z=a}$ ) and then reorganizing

$$
\begin{gathered}
-\left.\ell_{A} \omega_{z A}\right|_{z=a}-\left.\ell_{B} \omega_{z B}\right|_{z=a}=+\left.\ell_{A} \omega_{z A}\right|_{z=-a}+\left.\ell_{B} \omega_{z B}\right|_{z=-a} \\
\downarrow \\
-\left.\ell_{A} \omega_{z B}\right|_{z=-a}-\left.\ell_{B} \omega_{z B}\right|_{z=a}=\left.\ell_{A} \omega_{z B}\right|_{z=a}+\left.\ell_{B} \omega_{z B}\right|_{z=-a} \\
\downarrow \\
-\ell_{A}\left(\left.\omega_{z B}\right|_{z=-a}+\left.\omega_{z B}\right|_{z=a}\right)=+\ell_{B}\left(\left.\omega_{z B}\right|_{z=-a}+\left.\omega_{z B}\right|_{z=a}\right)
\end{gathered}
$$

Canceling out the vorticity term on both sides of the equation yields the length scale constraints for symmetrical flow

$$
\ell_{A}=-\ell_{B}
$$

The above (Eqs. 13-20) holds for spanwise symmetrical flow where the plane of symmetry is located at $\mathrm{z}=0$. In the above, each (sign of the) length-scale can only be applied to the associated analytic vorticity function on the same side of the plane of symmetry in which the length-scale is defined. In the context of the vortex filament reconnection problem, the non-symmetric flow length-scale constraint applies to the vortex breakdown for a filament with isolated breakpoint or an unbroken vortex filament; the symmetric flow length-scale constraint applies to the vortex filament reconnection process where the two breakpoints are relatively close proximity. The symmetric flow length-scale constraint also allows for the possibility of multiple vortex breakpoints with varying segment lengths, as along as spanwise symmetry is preserved. Finally, the above length scale relationship, though derived on the basis of axial velocity and vorticity symmetry, is also applicable to definition of the radial velocity. I.e., the following (Eq. 21) must also hold (for problems with symmetrical axial velocity and vorticity)

$$
v_{r}=-\ell_{A} \omega_{r A}-\ell_{B} \omega_{r B}
$$

Where 
For $t \rightarrow 0$

$$
\begin{array}{lll}
\omega_{r A}=0 & \text { When } & z \leq 0 \\
\omega_{r B}=0 & \text { When } & z \geq 0
\end{array}
$$

And for $t \rightarrow \infty$

$$
\begin{array}{lll}
\omega_{r A} \neq 0 & \text { When } & z \leq 0 \\
\omega_{r B} \neq 0 & \text { When } & z \geq 0
\end{array}
$$

Consequently, for this class of flow problems, where length scale factors can be applied, the unsteady viscous Helmholtz vorticity equation reduces to the well-known unsteady heat conduction equation. This analytical reduction of the vorticity equation to the unsteady heat conduction equation was previously demonstrated in Ref. 12. There is a large body of work related to the analytical solution of the heat conduction equation, including the use of unsteady finite-volume source modeling, that enabled the derivation of (albeit laminar) solutions for the vortex reconnection problem.

\section{Basic Framework for Analytical Solutions}

Given the above introductory discussion, the general solution approach for the vortex reconnection problem, assuming axisymmetric flow, is now outlined. This same solution approach was applied to all four initial vorticity distributions studied in this paper. Following this outline of the basic analytical framework, the four different vortex source terms used in the modeling of the vortex reconnection problem is presented. Because of space limitations only the full solution for the instantaneous vortex filament reconnection problem will be presented in the Appendix. However, as will be readily seen, the work presented in the Appendix is of general applicability to the other three initial vorticity distributions. The details of these other analytical solutions can be found in Ref. 12.

1. The axial vorticity distribution can be described by a semi-infinite continuous distribution of unsteady source terms:

$$
\begin{gathered}
\omega_{z}=\int_{s}^{\infty}\left(\left.\omega_{z}\right|_{\text {Source }}\right) d z_{*}+\int_{-\infty}^{-s}\left(\left.\omega_{z}\right|_{\text {Source }}\right) d z_{*} \\
\omega_{z A}=\int_{s}^{\infty}\left(\left.\omega_{z}\right|_{\text {Source }}\right) d z_{*} \\
\omega_{z B}=\int_{-\infty}^{-s}\left(\left.\omega_{z}\right|_{\text {Source }}\right) d z_{*}
\end{gathered}
$$

2. In turn, using length scale factors, the axial velocity can be determined from the axial vorticity; this is, in part, dependent on whether the flow is considered nonsymmetrical or symmetrical with respect to the filament axis.

Nonsymmetrical (Eqs. 9b and 11):

$$
v_{z}=-\ell \omega_{z}
$$

Symmetrical (Eqs. 17 and 20):

$$
v_{z}=-\ell\left(\omega_{z A}-\omega_{z B}\right)
$$


3. Given the above derivation of the axial velocity, the tangential velocity can be derived by means of the linear ordinary differential equation (derived from Eq. 5).

$$
\frac{\partial v_{\theta}}{\partial r}+\frac{v_{\theta}}{r}-\omega_{z}=0
$$

4. The radial vorticity can now be derived (based, again, on Eq. 5).

$$
\omega_{r}=-\frac{\partial v_{\theta}}{\partial z}
$$

If the flow is symmetrical, the radial vorticity expression will need to be further separated into the two terms, $\omega_{r A}$ and $\omega_{r B}$, such that

$$
\omega_{r}=\omega_{r A}+\omega_{r B}
$$

5. Next, the radial velocity, depending on whether the flow is considered symmetrical or nonsymmetrical, can be derived.

Nonsymmetrical (Eqs. 9a and 11):

$$
v_{r}=-\ell \omega_{r}
$$

Symmetrical (Eqs. 20 and 21):

$$
v_{r}=-\ell\left(\omega_{r A}-\omega_{r B}\right)
$$

5. Finally, the tangential vorticity can be derived to complete the solution for the unsteady, threedimensional vorticity/velocity flow components (derived from Eq. 5).

$$
\omega_{\theta}=\frac{\partial v_{r}}{\partial z}-\frac{\partial v_{z}}{\partial r}
$$

As noted above the solution of the vortex reconnection problem is dependent upon the type of source term used in the heat conduction equation. In this section four different source terms are briefly summarized that correspond to the four different initial vorticity distributions studied in this paper.

\section{A. Instantaneous Vortex Filaments (Point Source Term)}

Reference 16 provides a wealth of information regarding the solution of the heat conduction equation, including the use of point and line sources to solve for unsteady problems. Among the source terms identified in Ref. 16 is the well-known point source. Equation 22 employs point sources, Eq. 29, to define semi-infinite "instantaneous" vortex filament segment expressions for axial vorticity. 


$$
\left.\omega_{z}\right|_{\text {Source }}=\frac{\gamma}{8(\pi v t)^{3 / 2}} e^{-\left(r^{2}+\left(z-z_{*}\right)^{2}\right) / 4 v t}
$$

The complete vortex reconnection solution for instantaneous vortex filament segments is summarized in the Appendix.

\section{B. (Slightly) Parabolic Finite-Core Vortex Filaments}

Reference 16 provided exact expressions for spherical finite-volume source terms; one such expression is noted below

$$
\left.\omega_{z}\right|_{\text {Source }}=\frac{3 \gamma}{8 r_{o}^{3} \sqrt{\pi^{3} v t\left(r^{2}+\left(z-z_{*}\right)^{2}\right)}} \int_{0}^{r_{o}} r_{*}\left[e^{-\left(\sqrt{r^{2}+\left(z-z_{*}\right)^{2}}-r_{*}\right)^{2} / 4 v t}-e^{-\left(\sqrt{r^{2}+\left(z-z_{*}\right)^{2}}+r_{*}\right)^{2} / 4 v t}\right] d r_{*}
$$

Or, alternatively,

$$
\begin{aligned}
\left.\omega_{z}\right|_{\text {Source }}=\frac{3 \gamma}{8 \pi r_{o}^{3}} & \operatorname{erf}\left(\frac{\sqrt{r^{2}+\left(z-z_{*}\right)^{2}}+r_{o}}{2 \sqrt{v t}}\right)-\operatorname{erf}\left(\frac{\sqrt{r^{2}+\left(z-z_{*}\right)^{2}}-r_{o}}{2 \sqrt{v t}}\right) \\
& \left.-\frac{2 \sqrt{v t}}{\left.\sqrt{\pi\left(r^{2}+\left(z-z_{*}\right)\right.}\right)}\left[e^{-\left(\sqrt{r^{2}+\left(z-z_{*}\right)^{2}}-r_{o}\right)^{2} / 4 v t}-e^{-\left(\sqrt{r^{2}+\left(z-z_{*}\right)^{2}}+r_{o}\right)^{2} / 4 v t}\right]\right\}
\end{aligned}
$$

The parameter $r_{o}$ in Eqs. 30 and 31 is a spherical finite-volume source term initial "effective" radii. For the above source term, Eq. 30 or Eq. 31, $r_{o}$ is well defined, i.e. $\left.\omega_{z}\right|_{\text {Source }}=$ a nonzero constant for $r \leq r_{o}$ and $\left.\omega_{z}\right|_{\text {Source }}=0$ for $r>r_{o}$. Correspondingly, $r_{c 0}$ is an initial "finite core" vortex filament core radii, such that $\partial v_{\theta} /\left.\partial r\right|_{r=r_{c 0}}=0$ for $t=0$. In general, for finite-core vortices, with the notable exception of uniform-core vortices, the underlying source terms' initial "effective" radii is not equivalent to the initial vortex core size, i.e., in general, $r_{c 0} \neq r_{o}$. There is no a priori relationship between $r_{o}$ and $r_{c 0}$, except for the uniform-core (constant vorticity in the core) case where $r_{o}=r_{c 0}$. As it is anticipated that resulting parabolic-core vortex filament vorticity distribution will be nearly uniform, it is assumed - with the predictions in this paper made accordingly - that $r_{o} \approx r_{c 0}$. The validity of the resulting parabolic-core vortex filament analytic solution (as detailed in Ref. 12) is unaffected by this assumption other than a small effect on the spatial-temporal scaling of the predicted flow phenomena, e.g. as will be seen later the core size growth trend for the parabolic core solution is slightly under-predicted with respect to nondimensional time. 


\section{Dual-Core Vortex Filaments}

Reference 16 also introduced an "approximate" finite volume point source expression (Eq. 32). This finite volume source is approximation to the spherical finite volume source term noted in Eq. 31, valid for small values of $r_{o}$. This "approximate" source term is, as demonstrated in Ref. 12, an exact particular solution of the unsteady heat conduction equation. Therefore, though in one sense an approximation, it is perfectly valid to employ the Eq. 32 source term to define a "dual-core" vortex filament solution wherein the vortex has inner core of negative vorticity. Not unexpectedly, this dual-core solution has a lower limit, time-wise, as to its potential physical validity as singularities in the vorticity/circulation expressions are manifested as $t=0$. This dual-core model does exhibit predicted vortical flow characteristics that have analogous properties to real flow phenomena (e.g. variable vortex core axial distribution and the manifestation of vortex breakdown bubbles) and as such is included in this paper.

$$
\left.\omega_{z}\right|_{\text {Source }} \approx \frac{\gamma}{8(\pi v t)^{3 / 2}} e^{-\left(r^{2}+\left(z-z_{*}\right)^{2}\right) / 4 v t}\left\{1+\left[\frac{\left(r^{2}+\left(z-z_{*}\right)^{2}\right)}{v t}-6\right] \frac{r_{o}^{2}}{40 v t}\right\}
$$

Note that the parameter $r_{o}$ is still explicitly incorporated in the Eq. 32 source term expression because of its underlying heritage with respect to the Eq. 31 source term (of which it is a small core radius approximation thereof); though, as noted above, this parameter is indeterminate at its nominal defining initial condition because of the manifestation of a singularity in the source term at $t=0$. However, given the earlier proposed initial condition approximation, i.e. $r_{o} \approx r_{c 0}$, for the parabolic core vortex filament source term, an equivalent parameter value "assignment" logically can be made for the dual-core source term, i.e. $r_{o} \Rightarrow r_{c 0}$; by this means it is asserted that physically meaningful predictions can be made at all but the smallest values of nondimensional time.

\section{Uniform Core Vortex Filaments}

The following source term yields a solution wherein the vorticity radial distribution can be defined as initially uniform (versus the slightly parabolic initial distribution of the solution derived using spherical finite-volume sources). From Ref. 16, for the placement of the point sources at arbitrary polar-cylindrical coordinates (versus at $r=0$ for the "instantaneous" vortex filament segments), the following holds

$$
\left.\omega_{z}\right|_{\text {Source }}=\frac{\gamma r_{*}}{8 \sqrt{(\pi v t)^{3}}} e^{-\left\{\left(r^{2}+r_{*}^{2}-2 r r_{*} \cos \theta_{*}\right)+\left(z-z_{*}\right)^{2}\right\} / 4 v t}
$$

The above source term can be employed to define semi-infinite continuous line sources, subject to integration with respect to $\theta_{*}$ (from 0 to $2 \pi$ ) and $z_{*}$ (piecewise from $-s$ to $-\infty$ and $s$ to $\infty$ ), that then can be integrated with respect to $r_{*}$ (from 0 to $r_{c 0}$ ) to yield a uniform-core vortex filament solution. Equation 33 integration with respect to $\theta_{*}$ closely follows the work detailed in Ref. 16 and yields a modified Bessel function of the first kind of zero ${ }^{\text {th }}$ order, $I_{0}(x)$. Correspondingly, integration with respect to $z *$ yields results similar to the instantaneous vortex filament solution, which is detailed in the Appendix. The uniform-core solution is unaffected by the earlier assumed relationship, or rather equivalence, between $r_{o}$ and $r_{c 0}$. Complete solution details for the uniform-core vortex filament solution are provided in Ref. 12 . 
The resulting uniform-core solution has been found to have excellent agreement with an alternate related analytic treatment detailed in Ref. 17.

\section{Discussion of Results}

\section{A. Time-Dependent Behavior of Unbroken Vortices}

In addition to the well-known Lamb-Oseen vortex model, there are a number of alternate unsteady twodimensional vortex models noted in the literature - e.g. Refs. 12, 17, 18. Figure 6a-b compares the tangential velocity profiles, as a function of time, of the uniform finite-core solution (by way of illustration) to alternate unsteady vortex models cited in the literature. For example, the uniform finite-core solution is compared to both the Lamb-Oseen and Kirde (for two different parameter values of $n=1 / 2$ and $n=3 / 4$ ) vortex models. The Kirde solution (Ref. 18) is based, in part, on confluent hypergeometric functions. Figure 6a-b also compares the uniform finite core solution to the Aboelkassem, et al, unsteady, twodimensional Rankine-like vortex model (Ref. 17). (It should also be noted that the Ref. 17 methodology was later extended to other types of "monopolar" vortices, see Refs. 19-21.)

The Aboelkassem model and this paper's parabolic and uniform finite-core vortex solutions all transition from a Rankine-like initial tangential velocity profile to a Lamb-Oseen profile over time. The Aboelkassem vortex model is based on a Fourier-Bessel series formulation. The parameter values $n=1000$ (number of Bessel series terms) and $\beta=1 / 100$ (a multiplier constant) are used in the Aboelkassem model, as recommended. As the uniform finite core solution and the Aboelkassem vortex model both implicitly assume a Rankine vortex as an initial velocity profile, it is anticipated that there should be very good correlation between the two models, irrespective of their mathematical treatment/formulation. This is indeed the case. For all but the very earliest of nondimensional times, the uniform finite-core solution and the Aboelkassem models show excellent agreement. Both solutions have their strengths and weaknesses, though. The advantages of the Aboelkassem model is that it does not exhibit any numerical accuracy or stability issues throughout the complete range of solution applicability $\left(0 \leq t^{\bullet}<\infty\right)$. The main disadvantage of the Aboelkassem model is that a non-rigorous "calibration" of the Aboelkassem vortex model with the Lamb-Oseen velocity profile was performed (at a single point-wise nondimensional time, which, in Ref. 17, was somewhat arbitrarily selected) in order to define the recommended/suggested value for the parameter $\beta$. The uniform finite-core solution doesn't have to resort to such a mathematical artifice in its derivation. However, the uniform finite-core solution does exhibit numerical stability issues for very small values of time in its current mathematical formulation and numerical solution implementation. This can be partially compensated for by increasing the order of series terms included in the solution; the more proactive solution to the numerical stability issue would be to derive a specialized asymptotic formula for the uniform finite-core model for $t^{\bullet} \rightarrow 0$. 

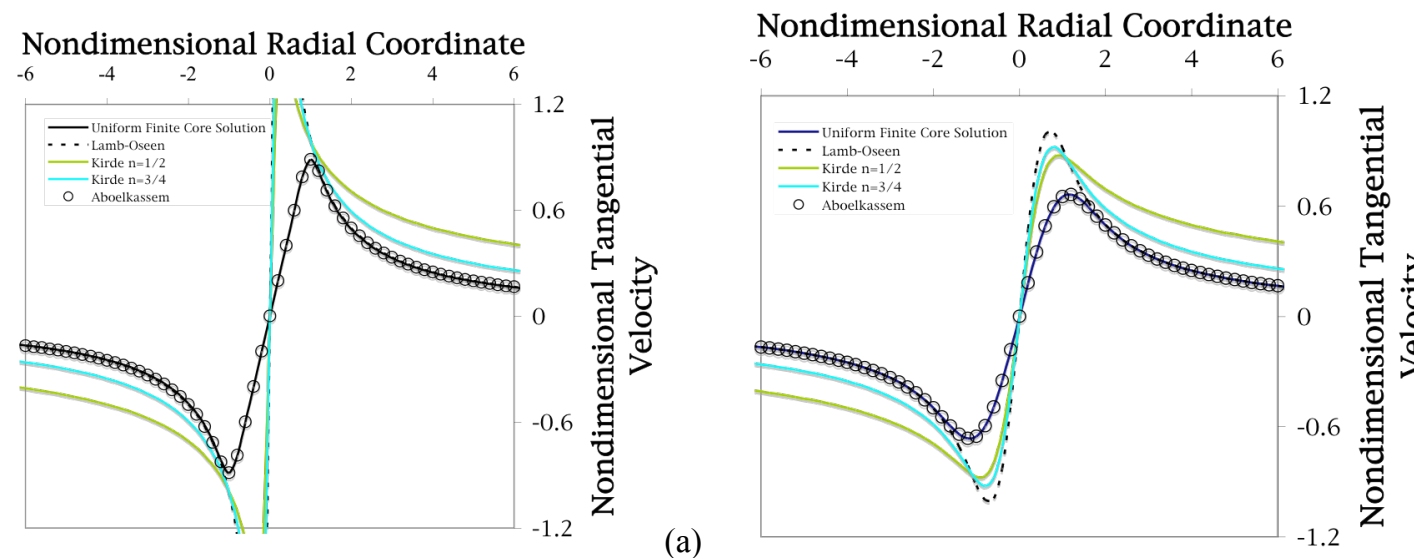

(b)

Fig. 6. Comparison of Unsteady Vortex Models: (a) $t^{\bullet}=0.01$ and (b) $t^{\bullet}=0.1$

The core radius growth (relative to the initial core size) with time for the various vortex models can be seen more clearly in Fig. 7a. The three vortex models (the Kirde ( $n=1 / 2$ and $n=3 / 4$ ), Aboelkassem, and uniform finite-core models) exhibit core radius growth with time as approximately $r_{c} \propto \sqrt{t}$. The Aboelkassem and uniform finite core models' core radius growth trends are reasonably close in agreement with the modified Lamb-Oseen core radius growth trend of $r_{c}=\sqrt{r_{c 0}^{2}+4 \alpha v t}$ where $\alpha=1.25643$ is the Lamb constant.

The uniform and parabolic finite-core tangential velocity profiles are nearly identical as discussed earlier (except at the earliest nondimensional times). The influence of the dual-core, parabolic, and uniform core models, with respect to core growth with time, is shown in Fig. 7b. As can also be seen in Fig. $7 \mathrm{~b}$ both the parabolic and dual-core vortex core size growth trends are under-predicted (the parabolic trend more so then the dual-core). This is likely a consequence of the "near-uniform-vorticity" assumption used in defining an "equivalence" between $r_{o}$ and $r_{c 0}$. This equivalence assumption will have to be reassessed in future work. Nonetheless, this issue does not fundamentally affect the overall validity of the parabolic and dual-core predictions (and underlying analytical solutions), it is instead primarily a spatiotemporal scaling issue and not a question of the basic flow phenomenology.

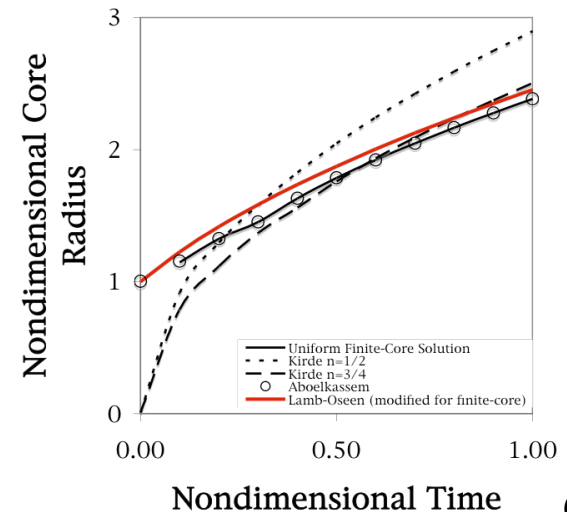

(a)

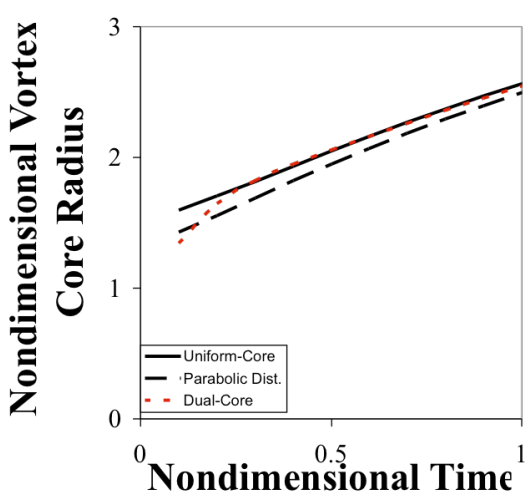

(b)

Fig. 7. Core Radius Growth Due to Viscous Diffusion: (a) Comparison to Models in Literature and (b) Comparison of Models Summarized in this Paper 


\section{B. Reconnection and Breakdown}

Figure $8 \mathrm{a}-\mathrm{b}$ shows the circulation distribution, along the filament axis, as a function of nondimensional time. The diffusion-like nature of the reconnection process is readily apparent in this figure as the vortex filament segments reconnect in the intermediate region between the vortex breakpoints. Secondary features in the circulation, though, can be seen resulting from the inner core of negative vorticity inherent in the dual-core initial vorticity distribution.
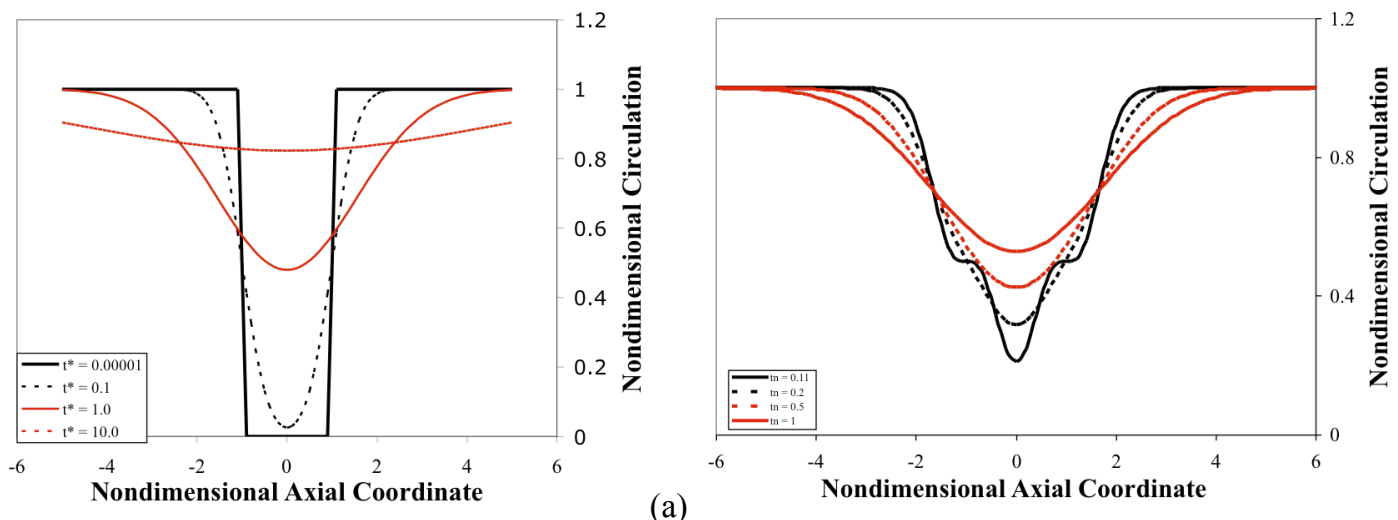

(a)

(b)

Fig. 8. Vortex Circulation Axial Distribution as a Function of Nondimensional Time: (a) "Instantaneous" and (b) Dual-Core Vortex Models

The secondary circulation distribution features observed in the Fig. 8b culminate in singularities (though not shown) at the vortex filament breakpoints as $t^{*} \rightarrow 0$. Therefore, a lower bound as to nondimensional time is required to prevent prediction of physically unrealistic flow. This lower bound was derived, in Ref. 12 , to be approximately $t^{*} \geq\left(r_{c 0}^{*}\right)^{2} / 20$.

Enstrophy is the mean square of all three vorticity components of the flow. Enstrophy is estimated by the expression $\eta \rightarrow\left(\omega_{z}^{2}+\omega_{r}^{2}+\omega_{\theta}^{2}\right) / 3$. In this particular set of flow problems, axial vorticity is the principal component for the enstrophy contours in Fig. 9a-c. The full complexity of the predicted vortex reconnection process can be seen in this figure. Presenting enstrophy contours for a sequential set of nondimensional times $\left(t^{*}=0.03\right.$ (parabolic-core only), 0.12, 0.2, 0.3, and 1.0) for one vortex-core-size-tocut ratio $\left(r_{c 0}^{*}=1.5\right)$ provides a powerful means of visualizing the vortex reconnection process. At the earliest nondimensional time values, the tangential and radial vorticity components contribute more significantly then at later time-wise. It is also at these early moments of the vortex reconnection process that clear differences can be distinguished between the enstrophy contour predictions of the three finitecore initial vorticity distributions. At later times, the enstrophy contours become nearly indistinguishable from each other. The axial flow wave front propagation boundaries can also be clearly seen in Fig. 9a-c as abrupt discontinuities in the enstrophy contours. 


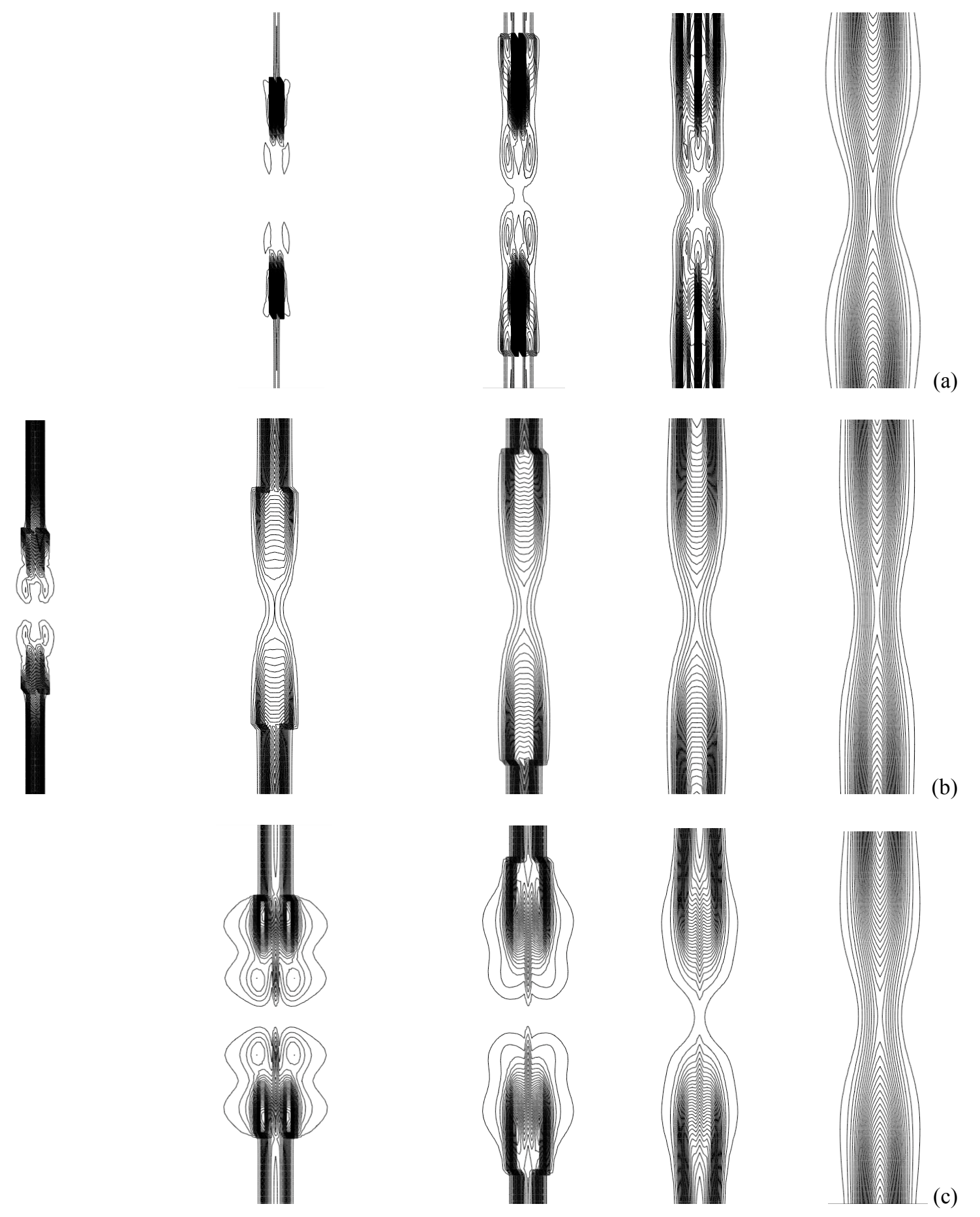

Fig. 9. Enstrophy $\left(r_{c 0}^{*}=1.5, \mathbf{R e}=\mathbf{5 0}\right.$, equivalent scaling, and at, sequentially, time values of $t^{*}=0.03$ (Parabolic-Core Only), $t^{*}=0.12, t^{*}=0.2, t^{*}=0.3$, and $\left.t^{*}=1.0\right)$ ): (a) dual-core solution, (b) parabolic-core, and (c) uniform-core

Figure 10 is a typical predicted velocity vector map (axial and radial velocities) superimposed over a color contour plot of the tangential (or swirl) velocity distribution in the intermediate region between the 
(two) "cut" vortex filament breakpoints. This velocity flow field, in the intermediate region between vortex filament breakpoints, is typical for all initial vorticity distributions studied.

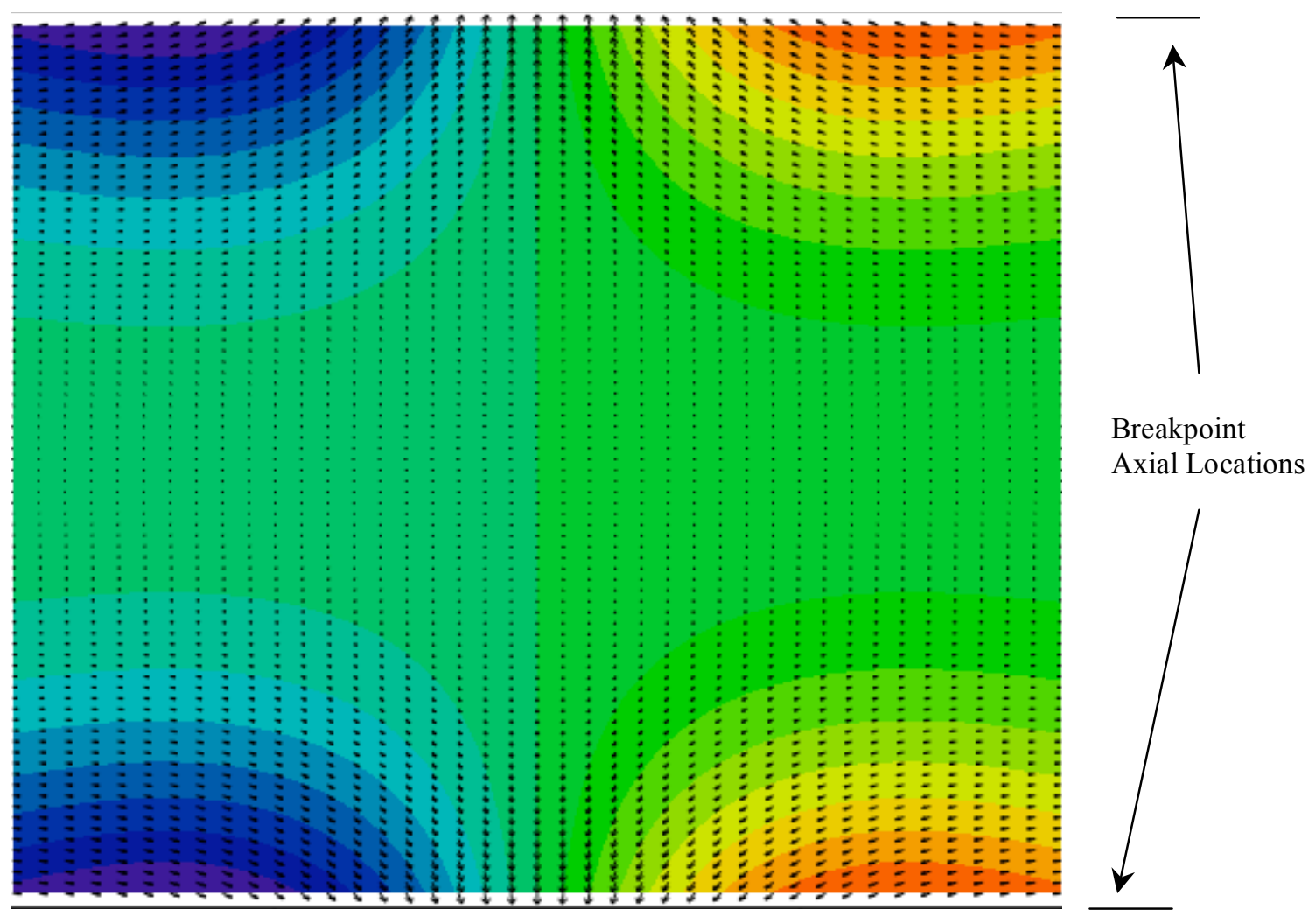

Fig. 10. Vector Field (vector components are $v_{r}$ and $v_{z} ; v_{\theta}$ indicated by color/shaded contours) for the $\mathbf{r} / \mathbf{z}$ Plane $\left(t^{*}=0.1\right)$

A universal flow feature of the vortex reconnection process appears to be the formation of the "funnellike" stream function contours and spiral streakline, or particle trace, flow patterns. Figure 11 presents a composite image of both the stream function contour and particle trace patterns for a typical result.

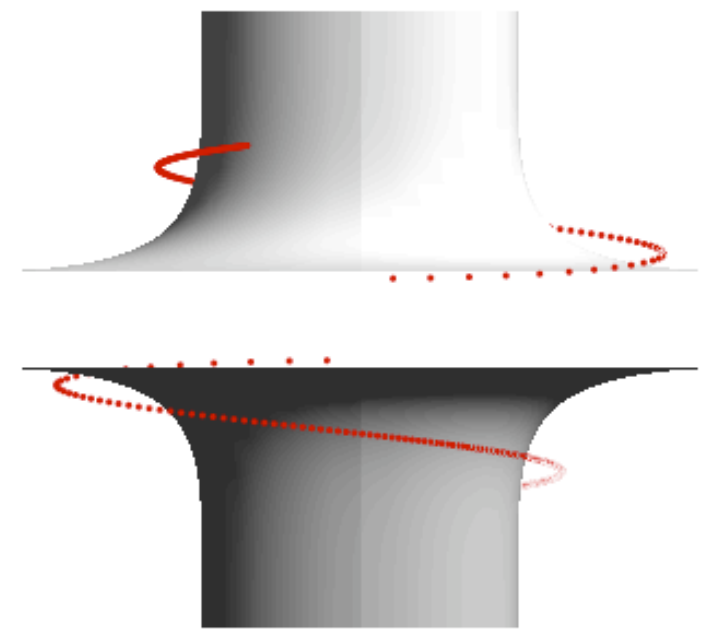

Fig. 11. Composite Image of Stream Function Iso-Surfaces $\left(t^{*}=1\right.$ and $\left.\mathbf{R e}=50\right)$ and Spiral Flow Pattern (Particle Trace between $1 \leq t^{*} \leq 100$ ) 
Both the parabolic and the dual-core initial vorticity distributions yielded predictions of variable core size distributions with respect to the axial coordinate (Figs. 12a-b and 13a-b). The dual-core predictions show greater variation in the core radius than does the parabolic initial vorticity distributions. It is interesting to observe that variable core radius distribution is not, as might be initially expected, solely a consequence of reduced or negative vorticity near the center of the vortex core, as the parabolic initial vorticity distribution also exhibits this flow feature. The under-prediction of the dual-core and parabolic core size in the far-field $\left(z^{*} \rightarrow \infty\right)$ for these relatively small nondimensional times can also be seen.
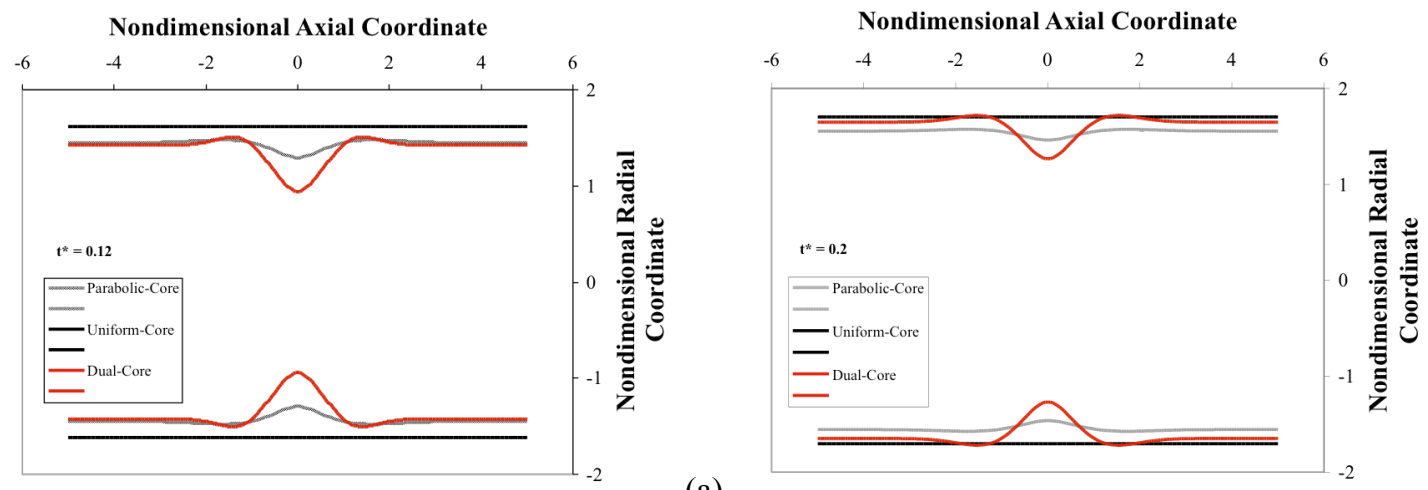

(a)

(b)

Fig. 12. Variable Vortex Core Size: (a) $t^{*}=0.12$ and (b) $t^{*}=0.2$
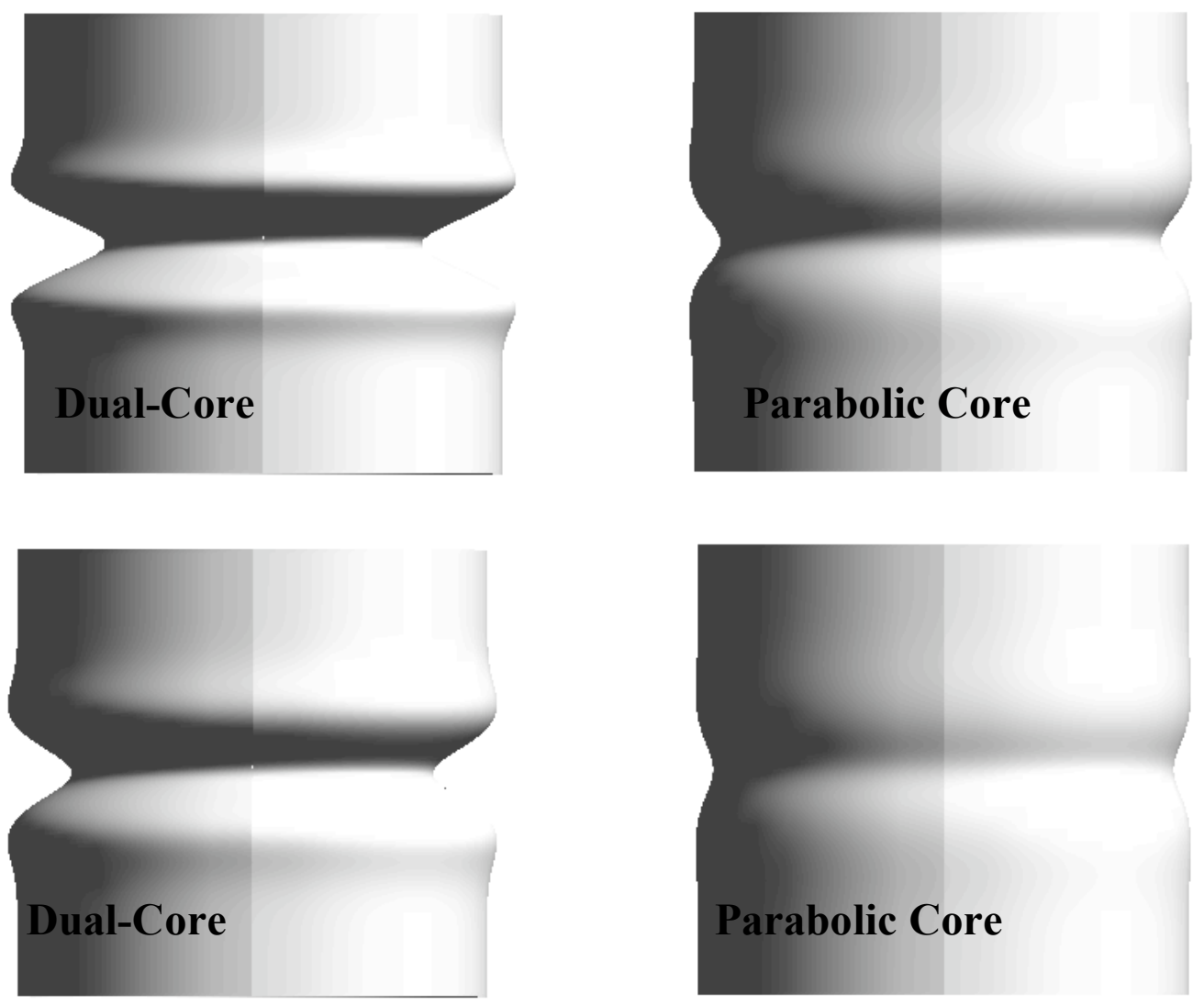

(a)

(b)

Fig. 13. Three Dimensional Contours of Variable Core Size: (a) $\left(t^{*}=0.12\right)$, and (b) $\left(t^{*}=0.2\right)$ 
As noted in Table 1, for the dual-core initial vorticity distribution, the vortex filament segments manifests flow features similar to that observed for vortex breakdown phenomena including the sudden expansion of the vortex core and the formation of recirculation bubbles. Figure 14a-d presents typical prediction results for the vortex core radius and breakdown bubble envelopes. Figure 15a-b goes further by illustrating the predicted vortex breakdown bubble geometry trend (as represented by both the bubble fineness ratio and the axial location, as a fraction of bubble length, of the maximum bubble thickness) as a function of nondimensional time and initial vortex core size. The predicted vortex breakdown bubble goes from being squat, acorn-like, to slender nearly spindle-like. Similar bubble topologies have been experimentally observed. Unique, perhaps, to the predicted vortex reconnection process, though, is that these vortex breakdown bubbles only occur in the early stages of the reconnection process and then dissipate/disappear after a critical nondimensional time value is reached; thereafter, the reconnection process is insensitive to the initial vorticity distribution and proceeds, in general, in a fairly similar manner.
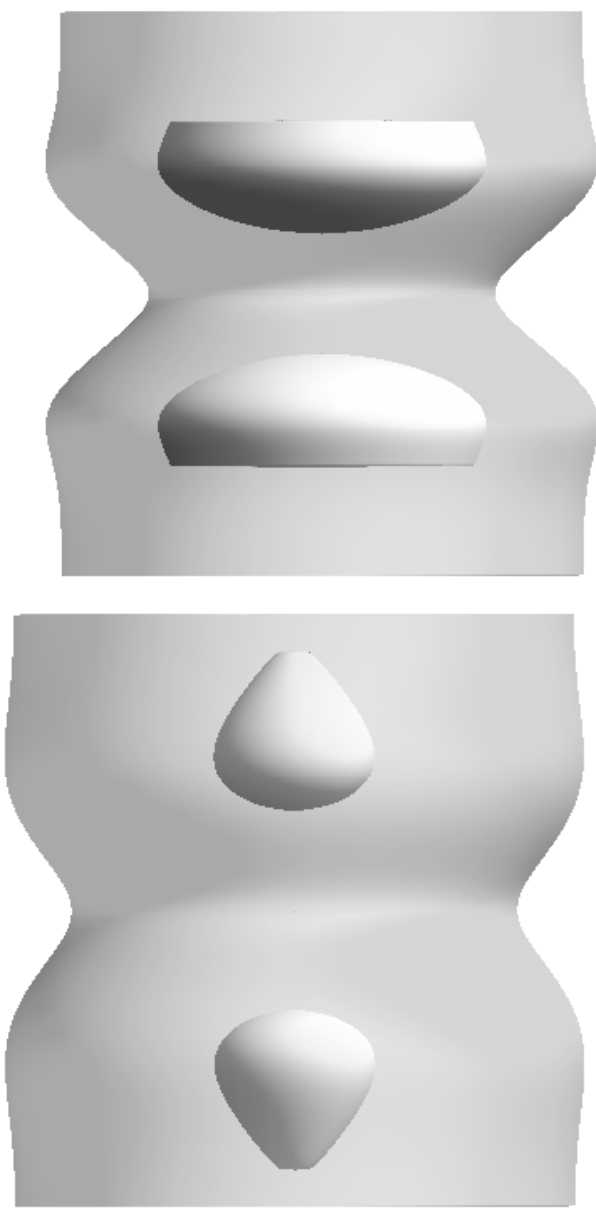

(c)

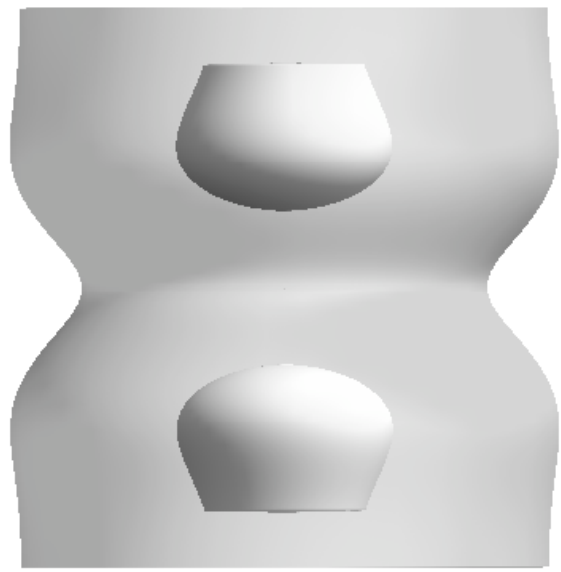

(a)

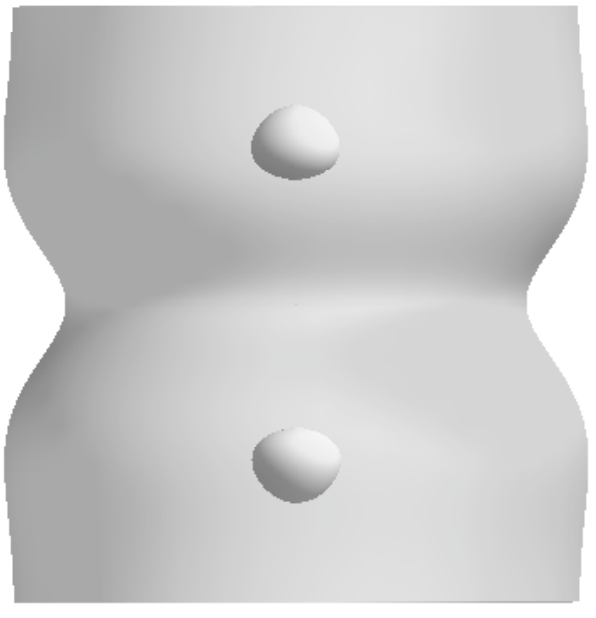

(b)

(d)

Fig. 14. Example of Predicted Vortex Core and Bubble Envelopes $\left(r_{c 0}^{*}=1.5\right.$ and $\left.\mathbf{R e}=\mathbf{5 0}\right)$ : (a) $t^{*}=0.12$, (b) $t^{*}=0.2$, (c) $t^{*}=0.23$, (d) $t^{*}=0.25$ 

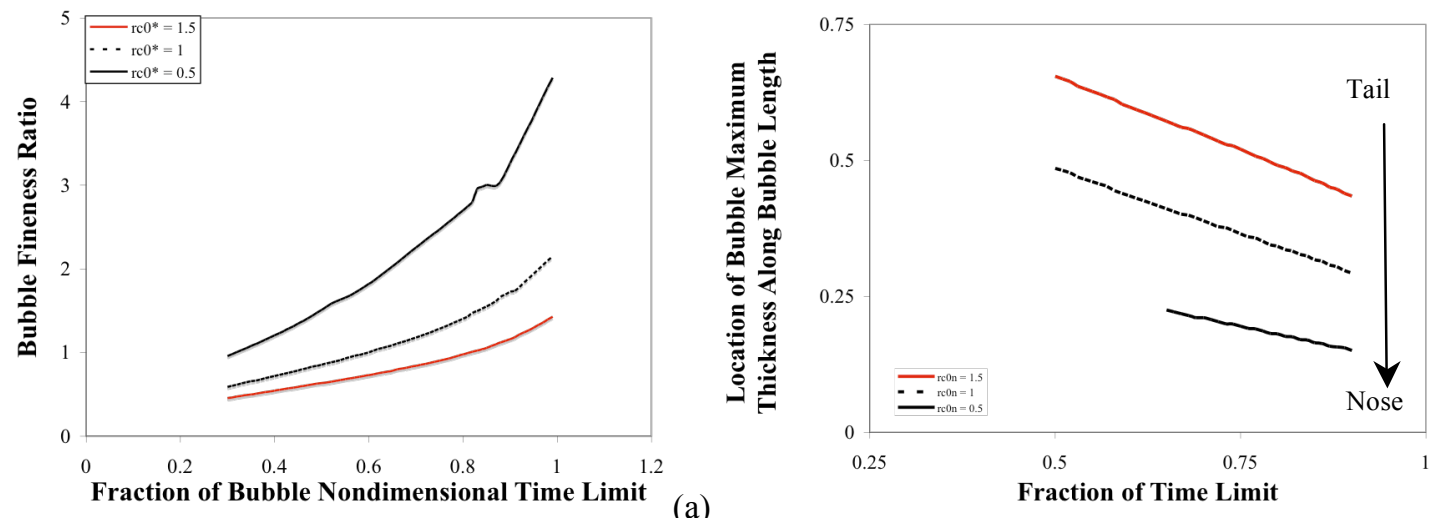

(b)

Fig. 15. Bubble Geometry Trend as a function of nondimensional time: (a) fineness ratio and (b) axial location (as a fraction of bubble length) of maximum thickness

Given the mathematic character of the dual-core vortex filament flow equations, it is relatively straightforward to identify discrete axisymmetric vortex breakdown bubble "finite states," and the nondimensional time limits that conform to them, that are exhibited in the predictions. Considerable discussion, including qualitative comparisons with experimental measurements and observations in the literature, is given in Ref. 12 as regards the prediction of this analogous flow behavior to the classic vortex breakdown phenomenon.

\section{Concluding Remarks}

Analytical solutions for the initial breakdown and subsequent reconnection of a laminar vortex filament - for the ideal case of having been "cut" or "broken" subsequent to a collision with a solid body -- has been developed. The analytical solutions do not treat the actual collision process itself, though. These analytical solutions belong to a general class of flow problems, identified through this work, that encompasses as a subset the extensively studied "moving boundary" laminar flow problem. The viscous Helmholtz equation for vorticity is reduced to the unsteady heat conduction equation though a novel treatment of the problem. Consequently, instantaneous and/or finite-core line sources for the vorticity can be used to model the unsteady flow vortex filament breakdown and reconnection processes. A diverse and fascinating catalog of flow features is predicted by the resulting analytical solution - including several examples of analog vortex breakdown flow structures (bubble and conical types) similar to that previously reported in the literature. The breadth of flow phenomenology predicted by the four initial vorticity distributions examined in this preliminary work begs continued investigation in this area, including a comprehensive examination of the influence of initial vorticity distributions on vortex evolution.

It is anticipated that the work presented in this paper will find future application to refined rotary- and fixed-wing computational wake models. Additionally, studies into active control/dissipation of vortical structures - such as trying to enhance the decay of trailed wing-tip vortices of large commercial aircraft to minimize the occurrence of wake upset for such aircraft - will also hopefully be furthered by this work. Finally, the general study of the vortex breakdown phenomena will also benefit from this work.

\section{References}

${ }^{1}$ Spencer, R.H., “Application of Vortex Visualization Test Techniques to Rotor Noise Research,” 26th Annual Forum of the American Helicopter Society, Philadelphia, PA, June 1970. 
${ }^{2}$ Fontana, R.R. and Hubbard, Jr., J.E., "A Comparison with Theory of Peak to Peak Sound Level for a Model Helicopter Rotor Generating Blade Slap at Low Tip Speeds," Ninth European Rotorcraft and Powered Lift Aircraft Forum, Stresa, Italy, September 1983.

${ }^{3}$ Swanson, A.A., "Application of the Shadowgraph Flow Visualization Technique to a Full-Scale Helicopter Rotor in Hover and Forward Flight," AIAA-93-3411, AIAA 11'th Applied Aerodynamics Conference, Monterey, CA, August, 1993.

${ }^{4}$ Light, J.S., Swanson, A.A., and Norman, T.R., "Application of the Wide-Field Shadowgraph Technique to Helicopters in Forward Flight," Journal of the American Helicopter Society, Vol. 37-No. 2, April 1992.

${ }^{5}$ Crouse Jr., G.L., Leishman, J.G., and Bi, N., "Theoretical and Experimental Study of Unsteady Rotor/Body Aerodynamic Interactions," Journal of the American Helicopter Society, Vol. 37-No. 1, January 1992.

${ }^{6}$ Foley, S.M., Funk, R.B., Fawcett, P.A., and Komerath, N.M., "Rotor-Wake-Induced Flow Separation on a Lifting Surface," Journal of the American Helicopter Society, Vol. 40-No. 2, April 1995.

${ }^{7}$ Brand, A., Komerath, N., and McMahon, H., "Results from Laser Sheet Visualization of a Periodic Rotor Wake," AIAA Journal of Aircraft, Vol. 26-No. 5, May 1989.

${ }^{8}$ Komerath, N., Wong, O., and Ganesh, B., "On the Formation and Decay of Rotor Blade Tip Vortices," AIAA 2004-2431, Fluid Mechanics Meeting, Portland, OR, June 2004.

${ }^{9}$ McCroskey, W.J., "Vortex Wakes of Rotorcraft," 33 ${ }^{\text {rd }}$ AIAA Aerospace Sciences Meeting, AIAA 95-0530, Reno, NV, January 9-12, 1995.

${ }^{10}$ Marshall, J.S., "Vortex Cutting by a Blade, Part 1: General Theory and a Simple Solution," AIAA Journal, Vol. 32, No. 6, June 1994.

${ }^{11}$ Weigand, A., "The Response of a Vortex Ring to a Transient Spatial Cut," Proceedings of the Seventh International Symposium on Flow Visualization, Ed. J. Crowder, Seattle, WA, September 1995.

${ }^{12}$ Young, L.A., "Vortex Flow Behavior Subsequent to Perpendicular Collision with a Solid Body," Soon to be Published NASA TM.

${ }^{13}$ Lamb, H., Hydrodynamics, Sixth Edition, Dover Publications, New York.

${ }^{14}$ White, F.M., Viscous Fluid Flow, McGraw-Hill, 1974.

${ }^{15}$ Saffman, P.G., Vortex Dynamics, Cambridge University Press, 1992.

${ }^{16}$ Carslaw, H.S. and Jaeger, J.C., Conduction of Heat in Solids, Second Edition, Clarendon Press, Oxford, 1959.

${ }^{17}$ Aboelkassem, Y., Vatistas, G.H., and Esmail, N., "Viscous Dissipation of Rankine Vortex Profile and Zero Meridional Flow,” Acta Mechanica Sinica, Vol. 21, No. 6, pp. 550-556.

${ }^{18}$ Kirde, K., "Untersuehungen über die zeitliche Weiterentwicklung eines Wirbels mit Vorgegebener Anfangsverteilung," Ingenieur-Arch, Vol. 31, 1962, pp. 385-404.

${ }^{19}$ Vatistas, G.H., Aboelkassem, Y., and Siddiqui, M.H.K., "On the Space-Time Duality of Intense Vortices," $35^{\text {th }}$ AIAA Fluid Dynamics Conference, Toronto, Canada, June 6-9, 2005.

${ }^{20}$ Vatistas, G.H., Aboelkassem, Y., and Siddiqui, M.H.K. "Time Decay of n Family of Vortices," AIAA Journal, Vol. 43, No. 6, June 2005.

${ }^{21}$ Vatistas, G.H. and Aboelkassem, Y., "Space-Time Analogy of Self-Similar Intense Vortices," AIAA Journal, Vol. 44, No. 4, April 2006.

${ }^{22}$ Beyer, W.H., Ed., CRC Standard Mathematical Tables, 26'th Edition, CRC Press, Boca Raton, Florida, 1981.

\section{Appendix - Instantaneous Vortex Filament Solution}

Reference 16 reveals that a particular solution of Eq. 4, for an instantaneous point source, is given by (Eq. 29 presented again for clarity)

$$
\left.\omega_{z}\right|_{\text {point source }}=\frac{c}{8(\pi v t)^{3 / 2}} e^{-\left(r^{2}+\left(z-z_{*}\right)^{2}\right) / 4 v t}
$$

Where $\mathrm{c}$ is a constant, $\mathrm{z} *$ is the origin of the instantaneous point source with respect to the $\mathrm{z}$-axis, and $\mathrm{r}$ and $\mathrm{z}$ are the coordinates of the point for which the vorticity is predicted.

Correspondingly, Ref. 16 derives the solution to the unsteady heat conduction equation for an instantaneous line source of infinite length (represented as a continuous distribution of instantaneous point sources) by Eq. 34 . 


$$
\begin{aligned}
\left.\omega_{z}\right|_{\text {infinite line source }} & =\frac{c}{8(\pi v t)^{3 / 2}} \int_{-\infty}^{\infty} e^{-\left(r^{2}+\left(z-z_{*}\right)^{2}\right) / 4 v t} d z_{*} \\
& =\frac{c}{4 \pi v t} e^{-r^{2} / 4 v t}
\end{aligned}
$$

With the constant, c, set equal to the circulation strength, $\gamma$, (Ref. 15), then Eq. 34 becomes the vorticity distribution for a Lamb-Oseen vortex.

To solve the unsteady laminar flow problem for a rectilinear vortex filament that has been "cut" or "broken," however, it is necessary to represent the vortex filament segments with semi-infinite distributions of instantaneous point sources (Eq. 35a-c). Note that the constant, $c$, has been assigned the value $c=\gamma$.

$$
\begin{aligned}
& \omega_{z}=\frac{\gamma}{8(\pi v t)^{3 / 2}} e^{-r^{2} / 4 v t}\left\{\int_{s}^{\infty} e^{-\left(z-z_{*}\right)^{2} / 4 v t} d z_{*}+\int_{-\infty}^{-s} e^{-\left(z-z_{*}\right)^{2} / 4 v t} d z_{*}\right\} \\
& \omega_{z A}=\frac{\gamma}{8(\pi v t)^{3 / 2}} e^{-r^{2} / 4 v t} \int_{s}^{\infty} e^{-(z-z *)^{2} / 4 v t} d z * \\
& \omega_{z B}=\frac{\gamma}{8(\pi v t)^{3 / 2}} e^{-r^{2} / 4 v t} \int_{-\infty}^{-s} e^{-\left(z-z_{*}\right)^{2} / 4 v t} d z_{*}
\end{aligned}
$$

$(35 \mathrm{a}-\mathrm{c})$

The integral terms in Eq. 35 can be found in standard handbooks for integration formulas (see Ref. 22). The solution of vorticity component with respect to the z-axis is given by

$$
\omega_{z}=\frac{\gamma}{8 \pi v t} e^{-r^{2} / 4 v t}\left\{\operatorname{erfc}\left(\frac{s-z}{\sqrt{4 v t}}\right)+\operatorname{erfc}\left(\frac{s+z}{\sqrt{4 v t}}\right)\right\}
$$

Where $\operatorname{erfc}(x)$ is the complementary error function, i.e.,

$$
\operatorname{erfc}(x)=\frac{2}{\sqrt{\pi}} \int_{x}^{\infty} e^{-u^{2}} d u
$$

Noting that complementary error function is related to the error function by the relationship $\operatorname{erfc}(x)=1-\operatorname{erf}(x)$. And, further, $\operatorname{erf}(-x)=-\operatorname{erf}(x), \operatorname{erf}(\infty)=1$, and $\operatorname{erf}(0)=0$. With these properties of the error function and complementary error function in mind, it can be readily seen that Eq. 36 satisfies three important boundary constraints and/or conditions.

$$
\omega_{z} \rightarrow \frac{\gamma}{4 \pi v t} e^{-r^{2} / 4 v t} \quad \text { For } \quad \text { (a) } s \rightarrow 0
$$


(b) $z \rightarrow \pm \infty$

Or when

$$
\text { (c) } t \rightarrow 0 \quad \text { And } \quad|z| \geq s
$$

The vorticity distribution approaches that of the Lamb-Oseen vortex as the above conditions (Eq. 38ac) are met or approached.

Having derived the vorticity distribution, the axial velocity distribution follows straightforwardly from Eq. 23b, given Eqs. 35-36.

$$
\begin{aligned}
v_{z} & =-\ell_{A} \omega_{z A}-\ell_{B} \omega_{z B} \\
& =-\ell\left(\omega_{z A}-\omega_{z B}\right)
\end{aligned}
$$

Where

$$
\begin{aligned}
& \omega_{z A}=\frac{\gamma}{8 \pi v t} e^{-r^{2} / 4 v t} \operatorname{erfc}\left(\frac{s-z}{\sqrt{4 v t}}\right) \\
& \omega_{z B}=\frac{\gamma}{8 \pi v t} e^{-r^{2} / 4 v t} \operatorname{erfc}\left(\frac{s+z}{\sqrt{4 v t}}\right)
\end{aligned}
$$

The appropriate length scale factor in Eq. 39a-c is the separation distance, specifically, $\ell \propto-s$. Besides being a natural length scale factor, using $\mathrm{s}$ as the length scale automatically satisfies the "moving boundary" condition of the vortex filament breakdown/reconnection problem; i.e., when $s=0$ then $v_{z}=0$. This choice for the length scale also satisfies that constraint that $v_{z} \geq 0$ for $z>0$ and $v_{z} \leq 0$ for $z<0$. However, the vortex filament solution also has to take into account a vortex axial flow "wave front" propagation boundary moving at a finite velocity, $\mathrm{V}_{\mathrm{P}}$, along the axis of the filament segments. Therefore, if $|z| \leq s+V_{P} t$ then $v_{z} \neq 0$; if $|z|>s+V_{P} t$ then $v_{z}=0$. It is especially important to note, though, that for the instantaneous vortex filament solution, this vortex axial flow wave front propagation occurs effectively instantaneously, i.e. $V_{P} \rightarrow \infty$, such that the axial velocity equation does not have to account for a wave front boundary cut-off.) Therefore, in general, the length scale now becomes $\ell=-s \cdot u\left(V_{P} t-(|z|-s)\right)$, where $u(x)$ is the unit (Heaviside) step function. An inherent assumption is that the "wave front" boundary axial location varies with time but not the radial coordinate. In general, the following definition for the wave front propagation velocity is employed

$$
\left.V_{P} \approx \frac{2}{r_{c 0}^{2}} \int_{0}^{\infty} v_{z} r d r\right|_{|z| \rightarrow \infty}
$$

Equation 40 assumes that the axial flow established by the vortex filament cut/break is primarily contained within the vortex core (which is only an approximation). Additionally, note that the integration of the axial velocity profile occurs behind and not ahead of the wave front. Specifically, though, noting the definition 
of the wave front propagation velocity, Eq. 40, that for the instantaneous vortex filament case $V_{P} \rightarrow \infty$ and, therefore, $\ell=-s$.

Application of the length scale factor to the axial vorticity expressions gives the following expression for the axial velocity

$$
v_{z}=\frac{\gamma s}{8 \pi v t} e^{-r^{2} / 4 v t}\left\{\operatorname{erfc}\left(\frac{s-z}{\sqrt{4 v t}}\right)-\operatorname{erfc}\left(\frac{s+z}{\sqrt{4 v t}}\right)\right\}
$$

Given Eqs. 24 and 36, the tangential velocity distribution can be derived through solution of a firstorder ordinary differential equation.

$$
\frac{d v_{\theta}}{d r}+P(r) v_{\theta}=Q(r)
$$

Where

$$
P(r)=1 / r
$$

And

$$
Q(r)=\frac{\gamma}{8 \pi v t}\left\{\operatorname{erfc}\left(\frac{s-z}{\sqrt{4 v t}}\right)+\operatorname{erfc}\left(\frac{s+z}{\sqrt{4 v t}}\right)\right\} \cdot e^{-r^{2} / 4 v t}
$$

Which has the general solution (Ref. 22)

$$
v_{\theta} e^{\int P(r) d r}=\int Q(r) e^{\int P(r) d r} d r+C
$$

Performing the required integration yields the expression

$$
v_{\theta}=\frac{1}{r}\left\{C-\frac{\gamma}{4 \pi}\left[\operatorname{erfc}\left(\frac{s-z}{\sqrt{4 v t}}\right)+\operatorname{erfc}\left(\frac{s+z}{\sqrt{4 v t}}\right)\right] e^{-r^{2} / 4 v t}\right\}
$$

The constant $\mathrm{C}$ (with respect to $\mathrm{r}$ ) can be found by imposing the constraint that $v_{\theta}=0$ for $\mathrm{r}=0$, for all $\mathrm{z}$ and t. Therefore,

$$
C=\frac{\gamma}{4 \pi}\left[\operatorname{erfc}\left(\frac{s-z}{\sqrt{4 v t}}\right)+\operatorname{erfc}\left(\frac{s+z}{\sqrt{4 v t}}\right)\right]
$$

And so the final solution for the vortex filament tangential velocity distribution is

$$
v_{\theta}=\frac{\gamma}{4 \pi r}\left(1-e^{-r^{2} / 4 v t}\right)\left\{\operatorname{erfc}\left(\frac{s-z}{\sqrt{4 v t}}\right)+\operatorname{erfc}\left(\frac{s+z}{\sqrt{4 v t}}\right)\right\}
$$


The analogous attributes of Eq. 46 with respect to the Lamb-Oseen vortex are obvious (Ref. 15). As demonstrated in a similar manner with respect to the vorticity distribution, if $s \rightarrow 0$ then, correspondingly, the tangential velocity distribution reduces to the Lamb-Oseen profile. Further, note that the vortex core radius is equivalent to that of a Lamb-Oseen vortex, i.e. $r_{c}=2.24 \sqrt{v t}$, and is invariant with respect to the filament break, in terms of $\mathrm{s}$, or the axial coordinate, $\mathrm{z}$, along the filament segments.

Next, given Eqs. 25, 27b, and 46, the radial vorticity, $\omega_{r}$, and velocity, $\mathrm{v}_{\mathrm{r}}$, can be derived by taking the derivative of $v_{\theta}$ with respect to $z$. Noting first, though, that the following holds true

$$
\frac{d}{d z} \operatorname{erfc}(x)=-\frac{2}{\sqrt{\pi}} e^{-x^{2}} \cdot \frac{d x}{d z}
$$

Therefore, applying the above formula for the derivative of the complementary error function the following results

$$
\omega_{r}=\frac{\gamma}{4 r \sqrt{\pi^{3} v t}}\left(1-e^{-r^{2} / 4 v t}\right)\left\{e^{-(s+z)^{2} / 4 v t}-e^{-(s-z)^{2} / 4 v t}\right\}
$$

And

$$
\begin{gathered}
\omega_{r A}=-\frac{\gamma}{4 r \sqrt{\pi^{3} v t}}\left(1-e^{-r^{2} / 4 v t}\right) e^{-(s-z)^{2} / 4 v t} \\
\omega_{r B}=+\frac{\gamma}{4 r \sqrt{\pi^{3} v t}}\left(1-e^{-r^{2} / 4 v t}\right) e^{-(s+z)^{2} / 4 v t}
\end{gathered}
$$

Applying the length scale factor, $\ell=-s$, to Eq. 48, given Eq. $27 \mathrm{~b}$ (presented below, again for clarity), such that

$$
\begin{aligned}
v_{r} & =-\ell_{A} \omega_{r A}-\ell_{B} \omega_{r B} \\
& =-\ell\left(\omega_{r A}-\omega_{r B}\right)
\end{aligned}
$$

This gives the following expression for the radial velocity distribution

$$
v_{r}=-\frac{\gamma s}{4 r \sqrt{\pi^{3} v t}}\left(1-e^{-r^{2} / 4 v t}\right)\left\{e^{-(s-z)^{2} / 4 v t}+e^{-(s+z)^{2} / 4 v t}\right\}
$$

A key outcome of Eq. 49 is the prediction that the radial velocity is negative (inward to the vortex core) for all values of $r, z$, and t. The radial inward flow contributes to the manifestation of "funnel-like" stream function contours and spiral (inward from the vortex breakpoints) streakline, or particle trace, flow patterns. This general vortex reconnection flow behavior holds true for all of the initial vorticity distributions studied in this paper. 